\title{
HOCHSCHILD COHOMOLOGY RELATED TO GRADED DOWN-UP ALGEBRAS WITH WEIGHTS $(1, n)$
}

\author{
AYAKO ITABA AND KENTA UEYAMA
}

\begin{abstract}
Let $A=A(\alpha, \beta)$ be a graded down-up algebra with $(\operatorname{deg} x, \operatorname{deg} y)=(1, n)$ and $\beta \neq 0$, and let $\nabla A$ be the Beilinson algebra of $A$. If $n=1$, then a description of the Hochschild cohomology group of $\nabla A$ is known. In this paper, we calculate the Hochschild cohomology group of $\nabla A$ for the case $n \geq 2$. As an application, we see that the structure of the bounded derived category of the noncommutative projective scheme of $A$ is different depending on whether $\left(\begin{array}{ll}1 & 0\end{array}\right)\left(\begin{array}{ll}\alpha & 1 \\ \beta & 0\end{array}\right)^{n}\left(\begin{array}{l}1 \\ 0\end{array}\right)$ is zero or not. Moreover, it turns out that there is a difference between the cases $n=2$ and $n \geq 3$ in the context of Grothendieck groups.
\end{abstract}

\section{INTRODUCTION}

Throughout let $k$ be an algebraically closed field of characteristic 0. A graded algebra

$$
A(\alpha, \beta):=k\langle x, y\rangle /\left(x^{2} y-\beta y x^{2}-\alpha x y x, x y^{2}-\beta y^{2} x-\alpha y x y\right) \quad \operatorname{deg} x=m, \operatorname{deg} y=n \in \mathbb{N}^{+}
$$

with parameters $\alpha, \beta \in k$ is called a graded down-up algebra. Down-up algebras were originally introduced by Benkart and Roby [4] in the study of the down and up operators on partially ordered sets. Since then, various aspects of these algebras have been investigated; for example, structures [5], [16, [23], representations [9], homological invariants [8], connections with enveloping algebras of Lie algebras [3], 4], invariant theory [14, [15], and so on. In particular, from the viewpoint of noncommutative projective geometry, the following property is of importance.

Theorem 1.1 ([16]). Let $A=A(\alpha, \beta)$ be a graded down-up algebra. Then $A$ is a noetherian AS-regular algebra of dimension 3 if and only if $\beta \neq 0$.

A graded down-up algebra has played a key role as a test case for more complicated situations in noncommutative projective geometry.

Let $A=\bigoplus_{i \in \mathbb{N}} A_{i}=A(\alpha, \beta)$ be a graded down-up algebra with $\beta \neq 0$, so that $A$ is AS-regular. Let $\ell=2(\operatorname{deg} x+\operatorname{deg} y)=2(m+n)$, so that $\ell$ is the Gorenstein parameter of $A$. Then the Beilinson algebra of $A$ is defined by

$$
\nabla A:=\left(\begin{array}{cccc}
A_{0} & A_{1} & \cdots & A_{\ell-1} \\
0 & A_{0} & \cdots & A_{\ell-2} \\
\vdots & \vdots & \ddots & \vdots \\
0 & 0 & \cdots & A_{0}
\end{array}\right)
$$

with the multiplication $\left(a_{i j}\right)\left(b_{i j}\right)=\left(\sum_{k=0}^{\ell-1} a_{k j} b_{i k}\right)$. For example, if $\operatorname{deg} x=1, \operatorname{deg} y=1$, then $\nabla A$ is isomorphic to the quotient of the path algebra of the quiver

$$
1 \underset{y_{1}}{\stackrel{x_{1}}{\rightleftharpoons}} 2 \underset{y_{2}}{\stackrel{x_{2}}{\rightleftharpoons}} 3 \underset{y_{3}}{\stackrel{x_{3}}{\rightleftharpoons}} 4
$$

modulo the ideal generated by the relations

$$
x_{1} x_{2} y_{3}-\beta y_{1} x_{2} x_{3}-\alpha x_{1} y_{2} x_{3}, \quad x_{1} y_{2} y_{3}-\beta y_{1} y_{2} x_{3}-\alpha y_{1} x_{2} y_{3} .
$$

2020 Mathematics Subject Classification. 16E40, 16S38, 16E05, $18 \mathrm{G} 80$.

Key words and phrases. Hochschild cohomology, down-up algebra, Beilinson algebra, derived equivalence. 
Let tails $A$ denote the quotient category of finitely generated graded right $A$-modules by the Serre subcategory of finite dimensional modules, and $\bmod \nabla A$ the category of finitely generated right $\nabla A$ modules. Note that tails $A$ is considered as the category of coherent sheaves on the noncommutative projective scheme associated to $A$ (see [1]). We write $\mathrm{D}^{\mathrm{b}}(\operatorname{tails} A)$ and $\mathrm{D}^{\mathrm{b}}(\bmod \nabla A)$ for the bounded derived categories of tails $A$ and $\bmod \nabla A$, respectively. The following is obtained as a special case of Minamoto-Mori's theorem [20, Theorem 4.14].

Theorem 1.2. If $A=A(\alpha, \beta)$ is a graded down-up algebra with $\beta \neq 0$, then $\nabla A$ is extremely Fano of global dimension 2 , and there exists an equivalence of triangulated categories

$$
\mathrm{D}^{\mathrm{b}}(\text { tails } A) \cong \mathrm{D}^{\mathrm{b}}(\bmod \nabla A) \text {. }
$$

We remark that a Fano algebra was renamed as an $n$-representation infinite algebra in [13] from the viewpoint of higher dimensional Auslander-Reiten theory. By Theorem 1.2, the Beilinson algebras of down-up algebras are important not only in noncommutative projective geometry but also in representation theory of finite dimensional algebras.

Our interest here is to study the Hochschild cohomology $\mathrm{HH}^{i}(\nabla A)$ of the Beilinson algebra $\nabla A$ of a down-up algebra $A$. It is known that the Hochschild cohomology of the Beilinson algebra of an AS-regular algebra $A$ is closely related to the Hochschild cohomology of tails $A$ and the infinitesimal deformation theory of tails $A$ (see [2], 18], [19]). In [2], Belmans computed the Hochschild cohomology of noncommutative planes and noncommutative quadrics, or in other words, the Hochschild cohomology of the Beilinson algebras of 3-dimensional quadratic or cubic AS-regular $\mathbb{Z}$-algebras. It should be noted that, to describe the Hochschild cohomology, he used a geometric technique based on the classification of the point schemes of 3-dimensional AS-regular algebras. Since the point schemes of down-up algebras are divided into three cases $\left(\mathbb{P}^{1} \times \mathbb{P}^{1}\right.$, a double curve of bidegree $(1,1)$, or two curves of bidegree $(1,1)$ in general position), [2, Table 2] implies the following result.

Theorem 1.3. Let $A=A(\alpha, \beta)$ be a graded down-up algebra with $\operatorname{deg} x=\operatorname{deg} y=1$ and $\beta \neq 0$. Then - $\operatorname{dim}_{k} \mathrm{HH}^{0}(\nabla A)=1$;

- $\operatorname{dim}_{k} \operatorname{HH}^{1}(\nabla A)= \begin{cases}6 & \text { if } \alpha=0, \\ 3 & \text { if } \alpha \neq 0 \text { and } \alpha^{2}+4 \beta=0, \\ 1 & \text { if } \alpha \neq 0 \text { and } \alpha^{2}+4 \beta \neq 0\end{cases}$

- $\operatorname{dim}_{k} \operatorname{HH}^{2}(\nabla A)= \begin{cases}9 & \text { if } \alpha=0, \\ 6 & \text { if } \alpha \neq 0 \text { and } \alpha^{2}+4 \beta=0, \\ 4 & \text { if } \alpha \neq 0 \text { and } \alpha^{2}+4 \beta \neq 0\end{cases}$

- $\operatorname{dim}_{k} \mathrm{HH}^{i}(\nabla A)=0$ for $i \geq 3$.

It is natural to ask what happens when $A$ is not generated in degree 1, i.e., how the structure of $\mathrm{HH}^{i}(\nabla A)$ depends on the grading of $A$. (If $A$ is a graded down-up algebra with $\operatorname{deg} x=m, \operatorname{deg} y=n$ such that $\operatorname{gcd}(m, n)=r$, then the $r$-th Veronese algebra $A^{(r)}$ is a graded down-up algebra with $\operatorname{deg} x=$ $m / r, \operatorname{deg} y=n / r$, and $\mathrm{HH}^{i}(\nabla A) \cong \mathrm{HH}^{i}\left(\nabla A^{(r)}\right)^{r}$ by [22, Theorem 9.1.8(1)], so it is enough to consider the case that $\operatorname{gcd}(\operatorname{deg} x, \operatorname{deg} y)=1$.) In this paper, we devote to compute $\operatorname{HH}^{i}(\nabla A)$ when $A$ is a graded down-up algebra with $\operatorname{deg} x=1, \operatorname{deg} y=n \geq 2(\operatorname{so}$ that $\operatorname{gcd}(\operatorname{deg} x, \operatorname{deg} y)=1$ and $\operatorname{deg} y$ is a multiple of $\operatorname{deg} x$ ). We will show the following theorem.

Theorem 1.4. Let $A=A(\alpha, \beta)$ be a graded down-up algebra with $\operatorname{deg} x=1, \operatorname{deg} y=n \geq 2$, and $\beta \neq 0$. We define

$$
\delta_{n}:=\left(\begin{array}{ll}
1 & 0
\end{array}\right)\left(\begin{array}{ll}
\alpha & 1 \\
\beta & 0
\end{array}\right)^{n}\left(\begin{array}{l}
1 \\
0
\end{array}\right) \in k
$$

(e.g. $\left.\delta_{2}=\alpha^{2}+\beta, \delta_{3}=\alpha^{3}+2 \alpha \beta, \delta_{4}=\alpha^{4}+3 \alpha^{2} \beta+\beta^{2}, \delta_{5}=\alpha^{5}+4 \alpha^{3} \beta+3 \alpha \beta^{2}\right)$. Then

- $\operatorname{dim}_{k} \mathrm{HH}^{0}(\nabla A)=1$; 


$$
\begin{aligned}
& \cdot \operatorname{dim}_{k} \mathrm{HH}^{1}(\nabla A)=\left\{\begin{array}{l}
4 \quad \text { if } n \text { is odd and } \alpha=0\left(\text { in this case } \delta_{n}=0\right), \\
3 \quad \text { if } n \text { is odd, } \alpha \neq 0, \text { and } \delta_{n}=0, \text { or if } n \text { is even and } \delta_{n}=0, \\
2 \quad \text { if } \alpha^{2}+4 \beta=0\left(\text { in this case } \delta_{n} \neq 0\right), \\
1 \quad \text { if } \delta_{n} \neq 0 \text { and } \alpha^{2}+4 \beta \neq 0 ;
\end{array}\right. \\
& \bullet \operatorname{dim}_{k} \operatorname{HH}^{2}(\nabla A)= \begin{cases}8 & \text { if } n=2 \text { and } \delta_{2}=0, \\
7 & \text { if } n=2 \text { and } \alpha^{2}+4 \beta=0\left(\text { in this case } \delta_{2} \neq 0\right), \\
6 & \text { if } n=2, \delta_{2} \neq 0, \text { and } \alpha^{2}+4 \beta \neq 0, \\
n+5 \quad \text { if } n \text { is odd and } \alpha=0\left(\text { in this case } \delta_{n}=0\right), \\
n+4 \quad \text { if } n \text { is odd, } \alpha \neq 0, \text { and } \delta_{n}=0, \text { or if } n \geq 4 \text { is even and } \delta_{n}=0, \\
n+3 \quad \text { if } n \geq 3 \text { and } \alpha^{2}+4 \beta=0\left(\text { in this case } \delta_{n} \neq 0\right), \\
n+2 \quad \text { if } n \geq 3, \delta_{n} \neq 0, \text { and } \alpha^{2}+4 \beta \neq 0 ;\end{cases}
\end{aligned}
$$

- $\operatorname{dim}_{k} \mathrm{HH}^{i}(\nabla A)=0$ for $i \geq 3$.

Note that it is crucial for this result that $\operatorname{deg} y$ is a multiple of $\operatorname{deg} x$; see Remark 2.5. Since $A$ is not generated in degree 1 , the geometric theory of point schemes does not work naively in our case, so our proof of Theorem 1.4 is purely algebraic.

It is known that Hochschild cohomology is invariant under derived equivalence. Using Theorem 1.2 , we have the following consequence.

Corollary 1.5. Let $A=A(\alpha, \beta)$ and $A^{\prime}=A\left(\alpha^{\prime}, \beta^{\prime}\right)$ be graded down-up algebras with $\operatorname{deg} x=1$, $\operatorname{deg} y=$ $n \geq 1$, where $\beta \neq 0, \beta^{\prime} \neq 0$. If

$$
\delta_{n}=\left(\begin{array}{ll}
1 & 0
\end{array}\right)\left(\begin{array}{ll}
\alpha & 1 \\
\beta & 0
\end{array}\right)^{n}\left(\begin{array}{l}
1 \\
0
\end{array}\right)=0 \text { and } \delta_{n}^{\prime}=\left(\begin{array}{ll}
1 & 0
\end{array}\right)\left(\begin{array}{ll}
\alpha^{\prime} & 1 \\
\beta^{\prime} & 0
\end{array}\right)^{n}\left(\begin{array}{l}
1 \\
0
\end{array}\right) \neq 0
$$

then $\mathrm{D}^{\mathrm{b}}$ (tails $\left.A\right) \not \mathrm{D}^{\mathrm{b}}$ (tails $\left.A^{\prime}\right)$.

In the last section, we will apply our results to the study of Grothendieck groups. For a graded down-up algebra $A=A(\alpha, \beta)$ with $\operatorname{deg} x=1, \operatorname{deg} y=n$, and $\beta \neq 0$, we can observe that if $n=1,2$, then $\mathrm{D}^{\mathrm{b}}$ (tails $A$ ) behaves a bit like a geometric object (a smooth projective surface), but if $n \geq 3$, then $\mathrm{D}^{\mathrm{b}}$ (tails $A$ ) is not equivalent to the derived category of any smooth projective surface (Proposition 3.2).

\section{Proof of Theorem 1.4}

In this section, we present the proof of Theorem 1.4. Throughout this section, let $A=A(\alpha, \beta)$ be a graded down-up algebra with $\operatorname{deg} x=1, \operatorname{deg} y=n \geq 2$, and $\beta \neq 0$. Then $\Lambda:=\nabla A$ is given as the quotient of the path algebra of the quiver

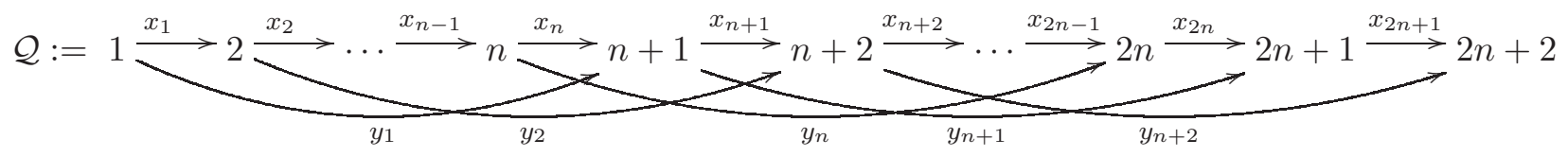

modulo the ideal generated by the relations

$$
\begin{aligned}
f_{i} & :=x_{i} x_{i+1} y_{i+2}-\beta y_{i} x_{i+n} x_{i+n+1}-\alpha x_{i} y_{i+1} x_{i+n+1} \quad(1 \leq i \leq n), \\
g & :=x_{1} y_{2} y_{n+2}-\beta y_{1} y_{n+1} x_{2 n+1}-\alpha y_{1} x_{n+1} y_{n+2} .
\end{aligned}
$$

Note that the arrows $x_{i}, y_{j}$ in $\mathcal{Q}$ come from the generators $x, y$ of $A$, respectively, and moreover, the relations $f_{i}, g$ come from the defining relations $x^{2} y-\beta y x^{2}-\alpha x y x, x y^{2}-\beta y^{2} x-\alpha y x y$ of $A$, respectively.

Let $\Lambda^{\mathrm{e}}:=\Lambda^{\mathrm{op}} \otimes \Lambda$ be the enveloping algebra of $\Lambda$. Then $\Lambda$ is a $\Lambda$-bimodule, or equivalently, a right $\Lambda^{\mathrm{e}}$-module. For $i \geq 0$, the $i$-th Hochschild cohomology group $\mathrm{HH}^{i}(\Lambda)$ of $\Lambda$ is defined by

$$
\operatorname{HH}^{i}(\Lambda):=\operatorname{Ext}_{\Lambda^{\mathrm{e}}}^{i}(\Lambda, \Lambda) .
$$


It is known that $\operatorname{HH}^{0}(\Lambda)$ coincides with the center of $\Lambda$. Since $\mathcal{Q}$ is connected and has no oriented cycles, we have

$$
\mathrm{HH}^{0}(\Lambda)=k
$$

(by a similar argument as in the proof of [17, Lemma 4.4]).

To compute $\mathrm{HH}^{i}(\Lambda)$ for $i \geq 1$, we first construct a minimal projective resolution of $\Lambda$ as a right $\Lambda^{\mathrm{e}}$-module by using Green-Snashall's method [10, Section 2]. We define the sets $\mathcal{G}^{i} \subset k \mathcal{Q}$ for $i=0,1,2$ by

$$
\mathcal{G}^{0}:=\left\{e_{1}, \ldots, e_{2 n+2}\right\}, \quad \mathcal{G}^{1}:=\left\{x_{1}, \ldots, x_{2 n+1}, y_{1}, \ldots, y_{n+2}\right\}, \quad \mathcal{G}^{2}:=\left\{f_{1}, \ldots, f_{n}, g\right\} .
$$

We set

$$
P^{i}:=\bigoplus_{h \in \mathcal{G}^{i}} \Lambda s(h) \otimes t(h) \Lambda \quad \text { for } i=0,1,2
$$

where $s(h)$ is the vertex at which $h$ starts (source) and $t(h)$ is the vertex at which $h$ ends (target). Then each $P^{i}$ is a projective right $\Lambda^{\mathrm{e}}$-module.

We define $\partial^{0}: P^{0} \rightarrow \Lambda$ to be the multiplication map, and $\partial^{1}: P^{1} \rightarrow P^{0}$ to be the right $\Lambda^{\mathrm{e}}$ homomorphism determined by

$$
\left\{\begin{array}{l}
s\left(x_{i}\right) \otimes t\left(x_{i}\right) \longmapsto\left(s\left(e_{i}\right) \otimes t\left(e_{i}\right)\right) x_{i}-x_{i}\left(s\left(e_{i+1}\right) \otimes t\left(e_{i+1}\right)\right), \\
s\left(y_{j}\right) \otimes t\left(y_{j}\right) \longmapsto\left(s\left(e_{j}\right) \otimes t\left(e_{j}\right)\right) y_{j}-y_{j}\left(s\left(e_{j+n}\right) \otimes t\left(e_{j+n}\right)\right)
\end{array}\right.
$$

for all $1 \leq i \leq 2 n+1,1 \leq j \leq n+2$. We also define $\partial^{2}: P^{2} \rightarrow P^{1}$ to be the right $\Lambda^{\mathrm{e}}$-homomorphism determined by

$$
\left\{\begin{aligned}
s\left(f_{i}\right) & \otimes t\left(f_{i}\right) \longmapsto \\
& \left(s\left(x_{i}\right) \otimes t\left(x_{i}\right)\right) x_{i+1} y_{i+2}+x_{i}\left(s\left(x_{i+1}\right) \otimes t\left(x_{i+1}\right)\right) y_{i+2}+x_{i} x_{i+1}\left(s\left(y_{i+2}\right) \otimes t\left(y_{i+2}\right)\right) \\
& -\beta\left(\left(s\left(y_{i}\right) \otimes t\left(y_{i}\right)\right) x_{i+n} x_{i+n+1}+y_{i}\left(s\left(x_{i+n}\right) \otimes t\left(x_{i+n}\right)\right) x_{i+n+1}+y_{i} x_{i+n}\left(s\left(x_{i+n+1}\right) \otimes t\left(x_{i+n+1}\right)\right)\right) \\
& -\alpha\left(\left(s\left(x_{i}\right) \otimes t\left(x_{i}\right)\right) y_{i+1} x_{i+n+1}+x_{i}\left(s\left(y_{i+1}\right) \otimes t\left(y_{i+1}\right)\right) x_{i+n+1}+x_{i} y_{i+1}\left(s\left(x_{i+n+1}\right) \otimes t\left(x_{i+n+1}\right)\right)\right), \\
s(g) & \otimes t(g) \longmapsto \\
& \left(s\left(x_{1}\right) \otimes t\left(x_{1}\right)\right) y_{2} y_{n+2}+x_{1}\left(s\left(y_{2}\right) \otimes t\left(y_{2}\right)\right) y_{n+2}+x_{1} y_{2}\left(s\left(y_{n+2}\right) \otimes t\left(y_{n+2}\right)\right) \\
& -\beta\left(\left(s\left(y_{1}\right) \otimes t\left(y_{1}\right)\right) y_{n+1} x_{2 n+1}+y_{1}\left(s\left(y_{n+1}\right) \otimes t\left(y_{n+1}\right)\right) x_{2 n+1}+y_{1} y_{n+1}\left(s\left(x_{2 n+1}\right) \otimes t\left(x_{2 n+1}\right)\right)\right) \\
& -\alpha\left(\left(s\left(y_{1}\right) \otimes t\left(y_{1}\right)\right) x_{n+1} y_{n+2}+y_{1}\left(s\left(x_{n+1}\right) \otimes t\left(x_{n+1}\right)\right) y_{n+2}+y_{1} x_{n+1}\left(s\left(x_{n+2}\right) \otimes t\left(x_{n+2}\right)\right)\right)
\end{aligned}\right.
$$

for all $1 \leq i \leq n$.

Lemma 2.1. With the above definitions, the sequence

$$
0 \longrightarrow P^{2} \stackrel{\partial^{2}}{\longrightarrow} P^{1} \stackrel{\partial^{1}}{\longrightarrow} P^{0} \stackrel{\partial^{0}}{\longrightarrow} \Lambda_{\Lambda} \mathrm{e} \longrightarrow 0
$$

forms a minimal projective resolution of $\Lambda$ as a right $\Lambda^{\mathrm{e}}$-module.

Proof. By construction, it follows from [10, Theorem 2.9] that $P^{2} \stackrel{\partial^{2}}{\longrightarrow} P^{1} \stackrel{\partial^{1}}{\longrightarrow} P^{0} \stackrel{\partial^{0}}{\longrightarrow} \Lambda \longrightarrow 0$ forms part of a minimal projective resolution. Since gldim $\Lambda=2$ by Theorem 1.2 , we see $\operatorname{pd}_{\Lambda^{\mathrm{e}}} \Lambda=2$ by [11, Lemma 1.5], so the third term, $P^{3}$, is 0 as required.

By applying the functor $\widehat{-}:=\operatorname{Hom}_{\Lambda^{\mathrm{e}}}(-, \Lambda)$ to (2.2), we have the Hochschild complex

$$
0 \longrightarrow \widehat{P^{0}} \stackrel{\widehat{\partial^{1}}}{\longrightarrow} \widehat{P^{1}} \stackrel{\partial^{2}}{\longrightarrow} \widehat{P^{2}} \longrightarrow 0 \text {. }
$$

We next calculate a $k$-basis of $\widehat{P^{i}}$. For $e_{i} \in \mathcal{G}^{0}$, we define the right $\Lambda^{\mathrm{e}}$-homomorphism $\tau_{e_{i}}: P^{0} \rightarrow \Lambda$ by

$$
\tau_{e_{i}}(s(h) \otimes t(h))= \begin{cases}e_{i} & \text { if } h=e_{i}, \\ 0 & \text { otherwise }\end{cases}
$$

for $h \in \mathcal{G}^{0}$. 
For $x_{i}, y_{j} \in \mathcal{G}^{1}$, we define the right $\Lambda^{\mathrm{e}}$-homomorphisms $\tau_{x_{i}}, \tau_{y_{j}}, \tau_{y_{j}}^{x^{n}}: P^{1} \rightarrow \Lambda$ by

$$
\begin{aligned}
& \tau_{x_{i}}(s(h) \otimes t(h))=\left\{\begin{array}{ll}
x_{i} & \text { if } h=x_{i}, \\
0 & \text { otherwise, }
\end{array} \quad \tau_{y_{j}}(s(h) \otimes t(h))= \begin{cases}y_{j} & \text { if } h=y_{j}, \\
0 & \text { otherwise },\end{cases} \right. \\
& \tau_{y_{j}}^{x^{n}}(s(h) \otimes t(h))= \begin{cases}x_{j} \cdots x_{j+n-1} & \text { if } h=y_{j}, \\
0 & \text { otherwise }\end{cases}
\end{aligned}
$$

for $h \in \mathcal{G}^{1}$.

For $f_{i}$ and $g \in \mathcal{G}^{2}$, we define the right $\Lambda^{\mathrm{e}}$-homomorphisms

$$
\tau_{f_{i}}^{x^{n+2}}, \tau_{f_{i}}^{y x^{2}}, \tau_{f_{i}}^{x y x}, \tau_{g}^{x^{2 n+1}}, \tau_{g}^{y x^{n+1}}, \tau_{g}^{x y x^{n}}, \tau_{g}^{y^{2} x}, \tau_{g}^{y x y}: P^{2} \rightarrow \Lambda
$$

by

$$
\begin{aligned}
& \tau_{f_{i}}^{x^{n+2}}(s(h) \otimes t(h))= \begin{cases}x_{i} \cdots x_{i+n+1} & \text { if } h=f_{i}, \\
0 & \text { otherwise, }\end{cases} \\
& \tau_{f_{i}}^{x y x}(s(h) \otimes t(h))= \begin{cases}x_{i} y_{i+1} x_{i+n+1} & \text { if } h=f_{i}, \\
0 & \text { otherwise, }\end{cases} \\
& \tau_{g}^{y x^{n+1}}(s(h) \otimes t(h))= \begin{cases}y_{1} x_{n+1} \cdots x_{2 n+1} & \text { if } h=g, \\
0 & \text { otherwise, }\end{cases}
\end{aligned}
$$

for $h \in \mathcal{G}^{2}$.

When $n=2$, for $f_{i} \in \mathcal{G}^{2}$, we additionally define the right $\Lambda^{\mathrm{e}}$-homomorphism $\tau_{f_{i}}^{y^{2}}: P^{2} \rightarrow \Lambda$ by

$$
\tau_{f_{i}}^{y^{2}}(s(h) \otimes t(h))= \begin{cases}y_{i} y_{i+2} & \text { if } h=f_{i} \\ 0 & \text { otherwise }\end{cases}
$$

for $h \in \mathcal{G}^{2}$.

Lemma 2.2. (1) $\widehat{P^{0}}$ has a k-basis $\left\{\tau_{e_{1}}, \ldots, \tau_{e_{2 n+2}}\right\}$, so $\operatorname{dim}_{k} \widehat{P^{0}}=2 n+2$.

(2) $\widehat{P^{1}}$ has a $k$-basis $\left\{\tau_{x_{i}}, \tau_{y_{j}}, \tau_{y_{j}}^{x^{n}} \mid 1 \leq i \leq 2 n+1,1 \leq j \leq n+2\right\}$, so $\operatorname{dim}_{k} \widehat{P^{1}}=4 n+5$.

(3) If $n=2$, then $\widehat{P^{2}}$ has a k-basis $\left\{\tau_{f_{i}}^{x^{4}}, \tau_{f_{i}}^{y x^{2}}, \tau_{f_{i}}^{x y x}, \tau_{f_{i}}^{y^{2}}, \tau_{g}^{x^{5}}, \tau_{g}^{y x^{3}}, \tau_{g}^{x y x^{2}}, \tau_{g}^{y^{2} x}, \tau_{g}^{y x y} \mid 1 \leq i \leq 2\right\}$, so $\operatorname{dim}_{k} \widehat{P^{2}}=13$.

(4) If $n \geq 3$, then $\widehat{P^{2}}$ has a k-basis $\left\{\tau_{f_{i}}^{x^{n+2}}, \tau_{f_{i}}^{y x^{2}}, \tau_{f_{i}}^{x y x}, \tau_{g}^{x^{2 n+1}}, \tau_{g}^{y x^{n+1}}, \tau_{g}^{x y x^{n}}, \tau_{g}^{y^{2} x}, \tau_{g}^{y x y} \mid 1 \leq i \leq n\right\}$, so $\operatorname{dim}_{k} \widehat{P^{2}}=3 n+5$.

Proof. Since $\widehat{P^{i}} \cong \bigoplus_{h \in \mathcal{G}^{i}} \operatorname{Hom}_{\Lambda^{\mathrm{e}}}(\Lambda s(h) \otimes t(h) \Lambda, \Lambda) \cong \bigoplus_{h \in \mathcal{G}^{i}} s(h) \Lambda t(h)$ as $k$-vector spaces, if $\tau \in \widehat{P^{i}}$, then $\tau$ is given as $\sum_{h \in \mathcal{G}^{i}} \tau_{h}$, and $\tau_{h}(s(h) \otimes t(h))$ is described by a linear combination of basis elements of $s(h) \Lambda t(h)$, so the result follows.

We then compute a matrix presentation of $\widehat{\partial^{2}}$. For $i \geq 1$, we define $a_{i}, b_{i} \in k$ by

$$
\left(\begin{array}{l}
a_{i} \\
b_{i}
\end{array}\right)=\left(\begin{array}{ll}
\alpha & 1 \\
\beta & 0
\end{array}\right)^{i-1}\left(\begin{array}{l}
1 \\
0
\end{array}\right)
$$

Lemma 2.3. For any $i \geq 1$, the equality

$$
x^{i} y=b_{i} y x^{i}+a_{i} x y x^{i-1}
$$

holds in $A=A(\alpha, \beta)$. 
Proof. We prove this by induction. The case $i=1$ is clear. Since

$$
\begin{aligned}
x^{i} y & =x x^{i-1} y=x\left(b_{i-1} y x^{i-1}+a_{i-1} x y x^{i-2}\right) \\
& =b_{i-1} x y x^{i-1}+a_{i-1}\left(\beta y x^{2}+\alpha x y x\right) x^{i-2} \\
& =\beta a_{i-1} y x^{i}+\left(\alpha a_{i-1}+b_{i-1}\right) x y x^{i-1},
\end{aligned}
$$

it follows that $a_{i}=\alpha a_{i-1}+b_{i-1}$ and $b_{i}=\beta a_{i-1}$, so we get the result.

Let $L_{1}$ denote the $(2 n+2) \times(3 n+3)$ matrix

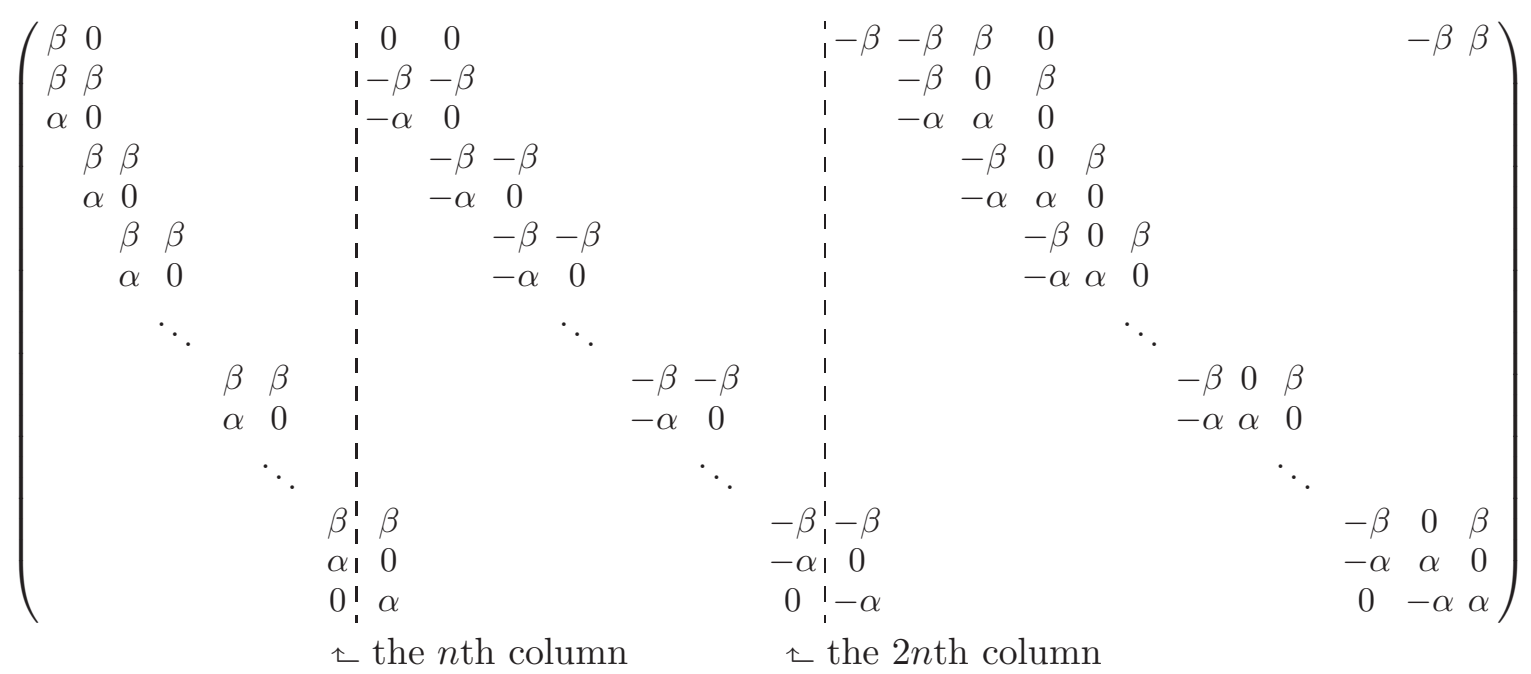

and let $L_{2}$ denote the $(n+2) \times(n+2)$ matrix

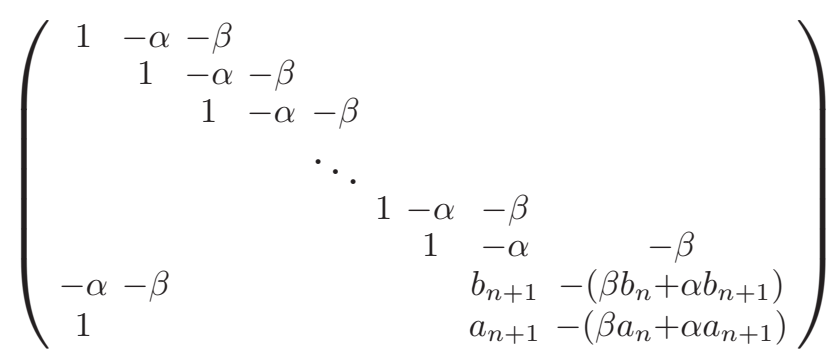

where the blank entries represent zeros.

By Lemma 2.3, $-\left(\beta b_{n}+\alpha b_{n+1}\right)=-\left(\beta b_{n}+\alpha \beta a_{n}\right)=-\beta a_{n+1}$, so we see

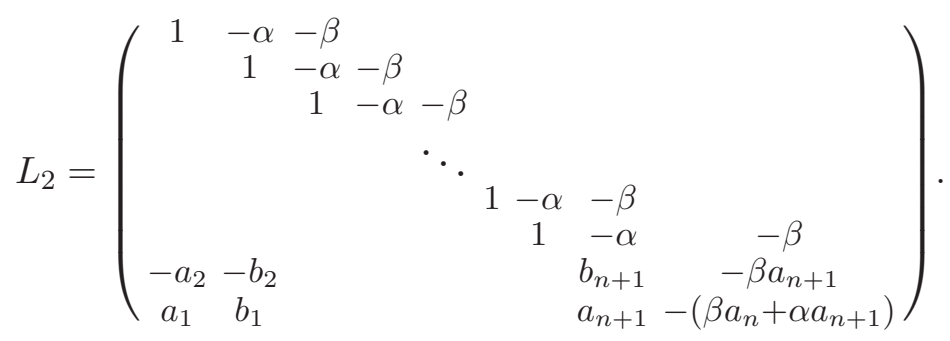

Lemma 2.4. (1) Assume that $n=2$. Let $\rho_{1}$ be the ordered basis $\left\{\tau_{x_{1}}, \ldots, \tau_{x_{5}}, \tau_{y_{1}}, \ldots, \tau_{y_{4}}, \tau_{y_{4}}^{x^{2}}, \ldots, \tau_{y_{1}}^{x^{2}}\right\}$ for $\widehat{P^{1}}$, and let $\rho_{2}$ be the ordered basis $\left\{\tau_{g}^{y^{2} x}, \tau_{f_{1}}^{y x^{2}}, \tau_{g}^{y x y}, \tau_{f_{2}}^{y x^{2}}, \tau_{f_{1}}^{x y x}, \tau_{f_{2}}^{x y x}, \tau_{f_{2}}^{x^{4}}, \tau_{f_{1}}^{x^{4}}, \tau_{g}^{y x^{3}}, \tau_{g}^{x y x^{2}}, \tau_{g}^{x^{5}}, \tau_{f_{1}}^{y^{2}}, \tau_{f_{2}}^{y^{2}}\right\}$ 
for $\widehat{P^{2}}$. Then the matrix representation $M_{2}$ of $\widehat{\partial^{2}}$ with respect to $\rho_{1}$ and $\rho_{2}$ is

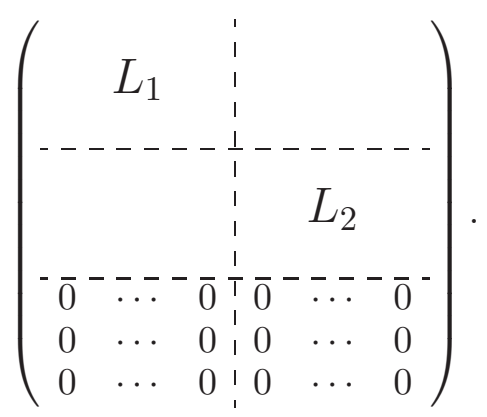

(2) Assume that $n \geq 3$. Let $\rho_{1}$ be the ordered basis $\left\{\tau_{x_{1}}, \ldots, \tau_{x_{2 n+1}}, \tau_{y_{1}}, \ldots, \tau_{y_{n+2}}, \tau_{y_{n+2}}^{x^{n}}, \ldots, \tau_{y_{1}}^{x^{n}}\right\}$ for $\widehat{P^{1}}$, and let $\rho_{2}$ be the ordered basis

$$
\left\{\tau_{g}^{y^{2} x}, \tau_{f_{1}}^{y x^{2}}, \tau_{g}^{y x y}, \tau_{f_{2}}^{y x^{2}}, \tau_{f_{1}}^{x y x}, \ldots, \tau_{f_{n}}^{y x^{2}}, \tau_{f_{n-1}}^{x y x}, \tau_{f_{n}}^{x y x}, \tau_{f_{n}}^{x^{n+2}}, \ldots, \tau_{f_{1}}^{x^{n+2}}, \tau_{g}^{y x^{n+1}}, \tau_{g}^{x y x^{n}}, \tau_{g}^{x^{2 n+1}}\right\}
$$

for $\widehat{P^{2}}$. Then the matrix representation $M_{2}$ of $\widehat{\partial^{2}}$ with respect to $\rho_{1}$ and $\rho_{2}$ is

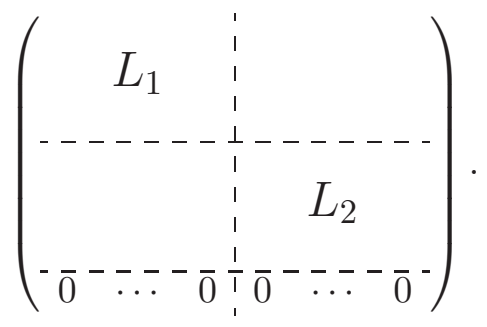

Proof. We prove only the last two columns of (2.3); the others and (1) are similar. First, for $h \in \mathcal{G}^{2}$, we have

$$
\begin{aligned}
& \widehat{\partial^{2}}\left(\tau_{y_{2}}^{x^{n}}\right)(s(h) \otimes t(h))=\left(\tau_{y_{2}}^{x^{n}} \circ \partial^{2}\right)(s(h) \otimes t(h)) \\
& =\left\{\begin{array}{ll}
\tau_{y_{2}}^{x^{n}}\left(-\beta\left(s\left(y_{2}\right) \otimes t\left(y_{2}\right)\right) x_{n+2} x_{n+3}+\cdots\right) & \text { if } h=f_{2}, \\
\tau_{y_{2}}^{x^{n}}\left(-\alpha x_{1}\left(s\left(y_{2}\right) \otimes t\left(y_{2}\right)\right) x_{n+2}+\cdots\right) & \text { if } h=f_{1}, \\
\tau_{y_{2}}^{x^{n}}\left(x_{1}\left(s\left(y_{2}\right) \otimes t\left(y_{2}\right)\right) y_{n+2}+\cdots\right) & \text { if } h=g,
\end{array} \quad= \begin{cases}-\beta x_{2} \cdots x_{n+1} x_{n+2} x_{n+3} & \text { if } h=f_{2}, \\
-\alpha x_{1} x_{2} \cdots x_{n+1} x_{n+2} & \text { if } h=f_{1}, \\
x_{1} x_{2} \cdots x_{n+1} y_{n+2} & \text { if } h=g .\end{cases} \right.
\end{aligned}
$$

Since $x_{1} x_{2} \cdots x_{n+1} y_{n+2}=b_{n+1} y_{1} x_{n+1} \cdots x_{2 n+1}+a_{n+1} x_{1} y_{2} x_{n+2} \cdots x_{2 n+1}$ by Lemma 2.3,

$$
\begin{aligned}
\widehat{\partial^{2}}\left(\tau_{y_{2}}^{x^{n}}\right)(s(h) \otimes t(h)) & = \begin{cases}-\beta x_{2} \cdots x_{n+1} x_{n+2} x_{n+3} & \text { if } h=f_{2} \\
-\alpha x_{1} x_{2} \cdots x_{n+1} x_{n+2} & \text { if } h=f_{1} \\
b_{n+1} y_{1} x_{n+1} \cdots x_{2 n+1}+a_{n+1} x_{1} y_{2} x_{n+2} \cdots x_{2 n+1} & \text { if } h=g\end{cases} \\
& =\left(-\beta \tau_{f_{2}}^{x^{n+2}}-\alpha \tau_{f_{1}}^{x^{n+2}}+b_{n+1} \tau_{g}^{y x^{n+1}}+a_{n+1} \tau_{g}^{x y x^{n}}\right)(s(h) \otimes t(h)),
\end{aligned}
$$

so we get the $(4 n+4)$ th column. Next, for $h \in \mathcal{G}^{2}$, we have

$$
\begin{aligned}
& \widehat{\partial^{2}}\left(\tau_{y_{1}}^{x^{n}}\right)(s(h) \otimes t(h))=\left(\tau_{y_{1}}^{x^{n}} \circ \partial^{2}\right)(s(h) \otimes t(h)) \\
& = \begin{cases}\tau_{y_{1}}^{x^{n}}\left(-\beta\left(s\left(y_{1}\right) \otimes t\left(y_{1}\right)\right) x_{n+1} x_{n+2}+\cdots\right) \\
\tau_{y_{1}}^{x^{n}}\left(-\beta\left(s\left(y_{1}\right) \otimes t\left(y_{1}\right)\right) y_{n+1} x_{2 n+1}-\alpha\left(s\left(y_{1}\right) \otimes t\left(y_{1}\right)\right) x_{n+1} y_{n+2}+\cdots\right) & \text { if } h=f_{1},\end{cases} \\
& = \begin{cases}-\beta x_{1} \cdots x_{n} x_{n+1} x_{n+2} & \text { if } h=f_{1}, \\
-\beta x_{1} \cdots x_{n} y_{n+1} x_{2 n+1}-\alpha x_{1} \cdots x_{n} x_{n+1} y_{n+2} & \text { if } h=g .\end{cases}
\end{aligned}
$$


It follows from Lemma 2.3 that

$$
\begin{aligned}
& -\beta x_{1} \cdots x_{n} y_{n+1} x_{2 n+1}-\alpha x_{1} \cdots x_{n} x_{n+1} y_{n+2} \\
& =-\beta\left(b_{n} y_{1} x_{n+1} \cdots x_{2 n}+a_{n} x_{1} y_{2} x_{n+2} \cdots x_{2 n}\right) x_{2 n+1}-\alpha\left(b_{n+1} y_{1} x_{n+1} \cdots x_{2 n+1}+a_{n+1} x_{1} y_{2} x_{n+2} \cdots x_{2 n+1}\right) \\
& =-\left(\beta b_{n}+\alpha b_{n+1}\right) y_{1} x_{n+1} \cdots x_{2 n+1}-\left(\beta a_{n}+\alpha a_{n+1}\right) x_{1} y_{2} x_{n+2} \cdots x_{2 n+1} .
\end{aligned}
$$

Thus we conclude

$$
\widehat{\partial^{2}}\left(\tau_{y_{1}}^{x^{n}}\right)(s(h) \otimes t(h))=\left(-\beta \tau_{f_{1}}^{x^{n+2}}-\left(\beta b_{n}+\alpha b_{n+1}\right) \tau_{g}^{y x^{n+1}}-\left(\beta a_{n}+\alpha a_{n+1}\right) \tau_{g}^{x y x^{n}}\right)(s(h) \otimes t(h))
$$

This gives the last column.

Remark 2.5. If $\operatorname{deg} x \leq \operatorname{deg} y$ and $\operatorname{deg} y$ is not a multiple of $\operatorname{deg} x$, then there are no paths of length $\operatorname{deg} y$ in the quiver of $\nabla A$, except for $y_{i}$ 's, so we see that "the $L_{2}$ part" does not appear in $M_{2}$.

Lemma 2.6. Let $\delta_{n}=\left(\begin{array}{ll}1 & 0\end{array}\right)\left(\begin{array}{ll}\alpha & 1 \\ \beta & 0\end{array}\right)^{n}\left(\begin{array}{l}1 \\ 0\end{array}\right)$.

(1) If $n$ is odd and $\alpha=0$, then $\delta_{n}=0$.

(2) If $\alpha^{2}+4 \beta=0$, then $\delta_{n}=(n+1)(\alpha / 2)^{n} \neq 0$.

Proof. (1) Clearly, $\delta_{1}=\alpha=0$. For $n \geq 3$,

$$
\delta_{n}=\left(\begin{array}{ll}
1 & 0
\end{array}\right)\left(\begin{array}{l}
a_{n+1} \\
b_{n+1}
\end{array}\right)=a_{n+1}=b_{n}=\beta a_{n-1}=\beta\left(\begin{array}{ll}
1 & 0
\end{array}\right)\left(\begin{array}{l}
a_{n-1} \\
b_{n-1}
\end{array}\right)=\beta \delta_{n-2}
$$

by Lemma 2.3 , so the result follows.

(2) If $\alpha^{2}+4 \beta=0$, then

$$
\left(\begin{array}{ll}
\alpha & 1 \\
\beta & 0
\end{array}\right)=\left(\begin{array}{cc}
\alpha & 1 \\
-\alpha^{2} / 4 & 0
\end{array}\right)=\left(\begin{array}{cc}
-1 & 1 \\
1+\alpha / 2 & -\alpha / 2
\end{array}\right)\left(\begin{array}{cc}
\alpha / 2 & 0 \\
1 & \alpha / 2
\end{array}\right)\left(\begin{array}{cc}
\alpha / 2 & 1 \\
1+\alpha / 2 & 1
\end{array}\right)
$$

so it follows that

$$
\left(\begin{array}{ll}
\alpha & 1 \\
\beta & 0
\end{array}\right)^{n}=\left(\begin{array}{cc}
-1 & 1 \\
1+\alpha / 2 & -\alpha / 2
\end{array}\right)\left(\begin{array}{cc}
(\alpha / 2)^{n} & 0 \\
n(\alpha / 2)^{n-1} & (\alpha / 2)^{n}
\end{array}\right)\left(\begin{array}{cc}
\alpha / 2 & 1 \\
1+\alpha / 2 & 1
\end{array}\right)
$$

Therefore, we have

$$
\delta_{n}=\left(\begin{array}{ll}
-1 & 1
\end{array}\right)\left(\begin{array}{cc}
(\alpha / 2)^{n} & 0 \\
n(\alpha / 2)^{n-1} & (\alpha / 2)^{n}
\end{array}\right)\left(\begin{array}{c}
\alpha / 2 \\
1+\alpha / 2
\end{array}\right)=(n+1)(\alpha / 2)^{n} .
$$

Since $\beta$ is nonzero, so is $\alpha$ and hence so is $\delta_{n}$.

Lemma 2.7. The rank of $L_{1}$ is $n$ if $n$ is odd and $\alpha=0$, and it is $n+1$ otherwise. 
Proof. Since $\beta \neq 0$, we have

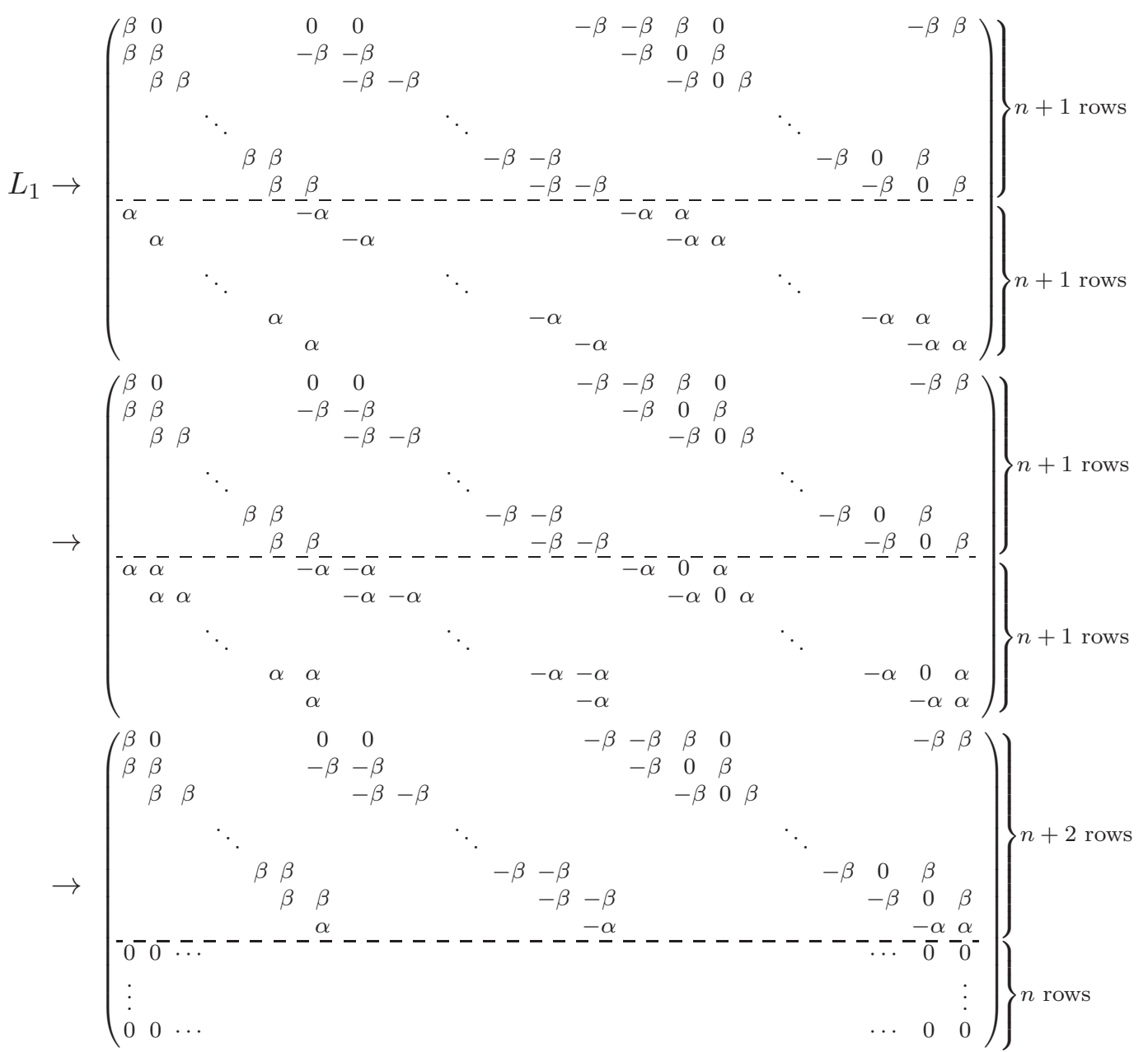

by elementary row operations, so we get

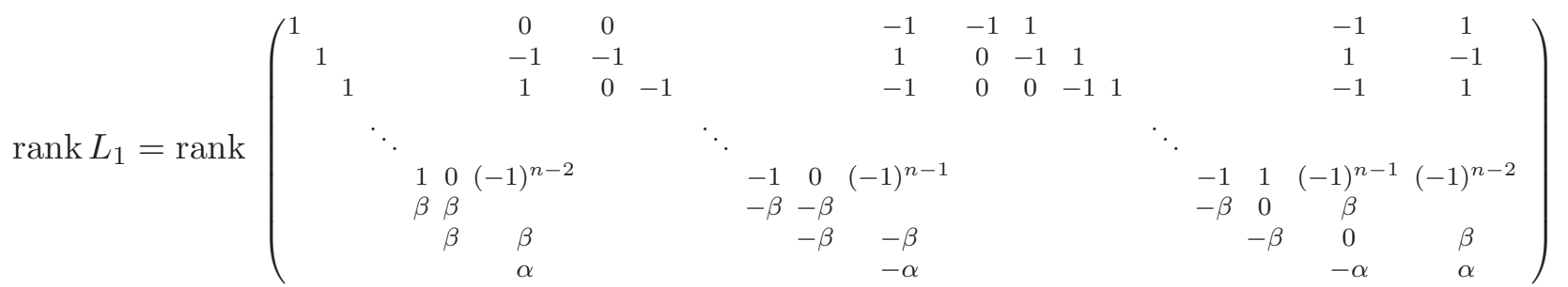

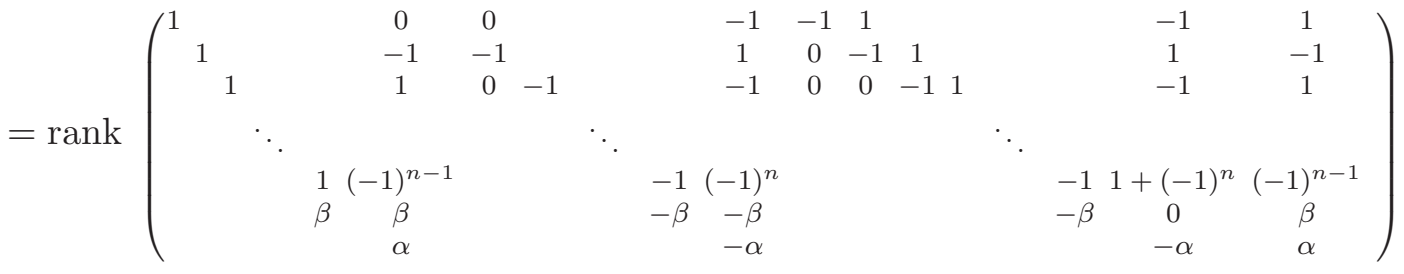

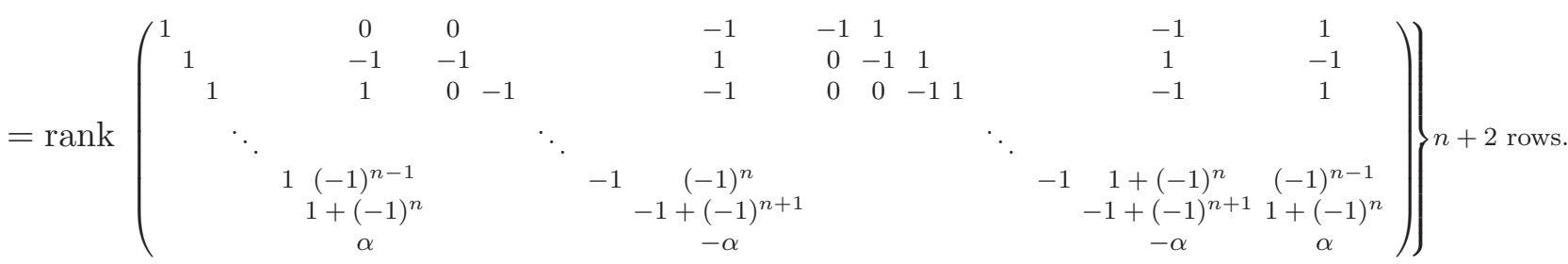


Hence the assertion follows.

Lemma 2.8. Let $\delta_{n}=\left(\begin{array}{ll}1 & 0\end{array}\right)\left(\begin{array}{ll}\alpha & 1 \\ \beta & 0\end{array}\right)^{n}\left(\begin{array}{l}1 \\ 0\end{array}\right)$. If $\delta_{n}=0$, then $\operatorname{rank} L_{2}=n$. If $\delta_{n} \neq 0$ and $\alpha^{2}+4 \beta=0$, then $\operatorname{rank} L_{2}=n+1$. If $\delta_{n} \neq 0$ and $\alpha^{2}+4 \beta \neq 0$, then $\operatorname{rank} L_{2}=n+2$.

Proof. By elementary row operations, we have

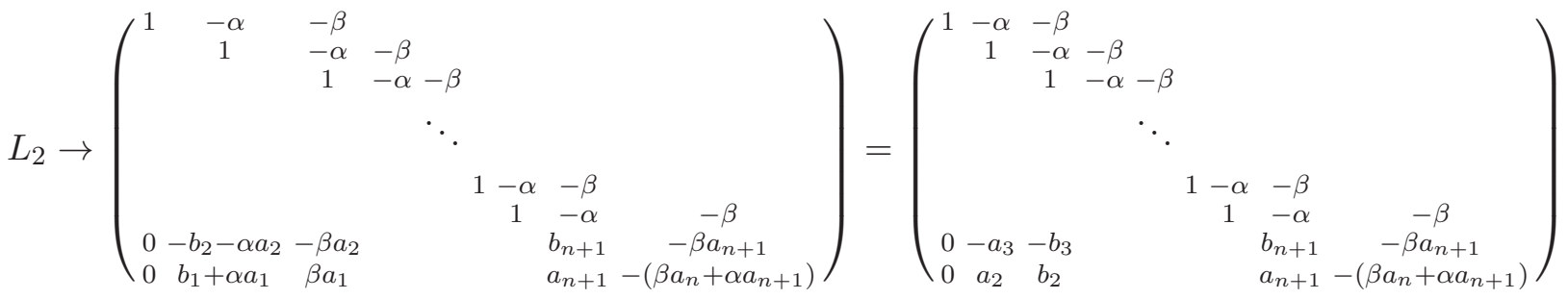

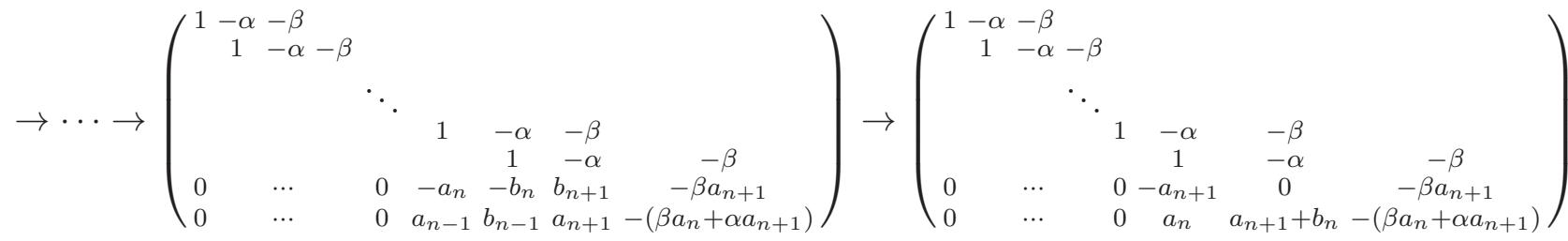

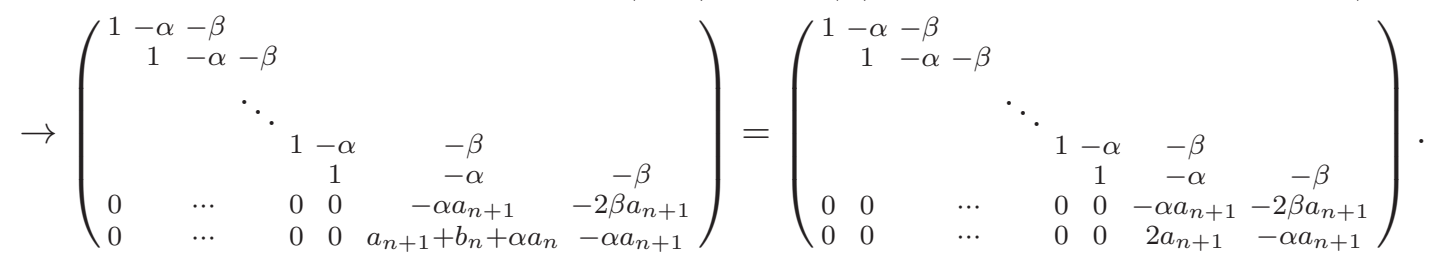

Since $\operatorname{det}\left(\begin{array}{cc}-\alpha a_{n+1} & -2 \beta a_{n+1} \\ 2 a_{n+1} & -\alpha a_{n+1}\end{array}\right)=a_{n+1}^{2}\left(\alpha^{2}+4 \beta\right)$ and $a_{n+1}=\left(\begin{array}{ll}1 & 0\end{array}\right)\left(\begin{array}{c}a_{n+1} \\ b_{n+1}\end{array}\right)=\delta_{n}$, the result follows.

We are now ready to prove Theorem 1.4 .

Proof of Theorem 1.4. By (2.1), we have $\operatorname{dim}_{k} \operatorname{HH}^{0}(\nabla A)=1$, and so $\operatorname{dim}_{k} \operatorname{Ker} \widehat{\partial^{1}}=1$. It follows from Lemma 2.2(1) that $\operatorname{dim}_{k} \operatorname{Im} \widehat{\partial^{1}}=\operatorname{dim}_{k} \widehat{P^{0}}-\operatorname{dim}_{k} \operatorname{Ker} \widehat{\partial^{1}}=(2 n+2)-1=2 n+1$. Lemmas 2.2 (2) and 2.4 imply

$$
\begin{aligned}
\operatorname{dim}_{k} \mathrm{HH}^{1}(\nabla A) & =\operatorname{dim}_{k} \operatorname{Ker} \widehat{\partial^{2}}-\operatorname{dim}_{k} \operatorname{Im} \widehat{\partial^{1}} \\
& =\left(\operatorname{dim}_{k} \widehat{P^{1}}-\operatorname{dim}_{k} \operatorname{Im} \widehat{\partial^{2}}\right)-\operatorname{dim}_{k} \operatorname{Im} \widehat{\partial^{1}} \\
& =\operatorname{dim}_{k} \widehat{P^{1}}-\operatorname{rank} M_{2}-\operatorname{dim}_{k} \operatorname{Im} \widehat{\partial^{1}} \\
& =(4 n+5)-\operatorname{rank} L_{1}-\operatorname{rank} L_{2}-(2 n+1) \\
& =2 n+4-\operatorname{rank} L_{1}-\operatorname{rank} L_{2} .
\end{aligned}
$$

By Lemmas 2.6, 2.7, and 2.8, we obtain

$\operatorname{dim}_{k} \mathrm{HH}^{1}(\nabla A)$

$$
= \begin{cases}2 n+4-n-n=4 & \text { if } \left.n \text { is odd and } \alpha=0 \text { (in this case } \delta_{n}=0\right), \\ 2 n+4-(n+1)-n=3 & \text { if } n \text { is odd, } \alpha \neq 0, \text { and } \delta_{n}=0, \text { or if } n \text { is even and } \delta_{n}=0, \\ 2 n+4-(n+1)-(n+1)=2 & \text { if } \left.\alpha^{2}+4 \beta=0 \text { (in this case } \delta_{n} \neq 0\right), \\ 2 n+4-(n+1)-(n+2)=1 & \text { if } \delta_{n} \neq 0 \text { and } \alpha^{2}+4 \beta \neq 0 .\end{cases}
$$


Moreover, since

$$
\begin{aligned}
\operatorname{dim}_{k} \mathrm{HH}^{2}(\nabla A) & =\operatorname{dim}_{k} \operatorname{Ker} \widehat{\partial^{3}}-\operatorname{dim}_{k} \operatorname{Im} \widehat{\partial^{2}} \\
& =\operatorname{dim}_{k} \widehat{P^{2}}-\operatorname{rank} M_{2} \\
& =\operatorname{dim}_{k} \widehat{P^{2}}-\operatorname{rank} L_{1}-\operatorname{rank} L_{2} \\
& =\operatorname{dim}_{k} \widehat{P^{2}}+\operatorname{dim}_{k} \operatorname{HH}^{1}(\nabla A)-(2 n+4) \\
& = \begin{cases}13+\operatorname{dim}_{k} \operatorname{HH}^{1}(\nabla A)-8 & \text { if } n=2, \\
3 n+5+\operatorname{dim}_{k} \operatorname{HH}^{1}(\nabla A)-(2 n+4) & \text { if } n \geq 3,\end{cases} \\
& = \begin{cases}\operatorname{dim}_{k} \operatorname{HH}^{1}(\nabla A)+5 & \text { if } n=2, \\
\operatorname{dim}_{k} \operatorname{HH}^{1}(\nabla A)+n+1 & \text { if } n \geq 3\end{cases}
\end{aligned}
$$

by Lemmas $2.2(3),(4)$, it follows that

$$
\operatorname{dim}_{k} \operatorname{HH}^{2}(\nabla A)=\left\{\begin{array}{l}
8 \text { if } n=2 \text { and } \delta_{2}=0, \\
7 \quad \text { if } n=2 \text { and } \alpha^{2}+4 \beta=0\left(\text { in this case } \delta_{2} \neq 0\right), \\
6 \quad \text { if } n=2, \delta_{2} \neq 0, \text { and } \alpha^{2}+4 \beta \neq 0, \\
\left.n+5 \text { if } n \text { is odd and } \alpha=0 \text { (in this case } \delta_{n}=0\right), \\
n+4 \quad \text { if } n \text { is odd, } \alpha \neq 0, \text { and } \delta_{n}=0, \text { or if } n \geq 4 \text { is even and } \delta_{n}=0, \\
\left.n+3 \text { if } n \geq 3 \text { and } \alpha^{2}+4 \beta=0 \text { (in this case } \delta_{n} \neq 0\right), \\
n+2 \quad \text { if } n \geq 3, \delta_{n} \neq 0, \text { and } \alpha^{2}+4 \beta \neq 0 .
\end{array}\right.
$$

Clearly $\mathrm{HH}^{i}(\nabla A)=0$ for $i \geq 3$, so the proof is completed.

\section{Discussion on Grothendieck Groups}

At the end of the paper, we give a discussion of our results in the context of Grothendieck groups based on [7, Section 3] and [3, Section 3.1]. Let $\mathrm{T}$ be a triangulated category, and let $K_{0}(\mathrm{~T})$ be the Grothendieck group of $\mathrm{T}$ (see [7, Section 3] for details). If $\mathrm{T}$ admits a full strong exceptional sequence of length $r$, then $K_{0}(\mathrm{~T})$ is $\mathbb{Z}^{r}$, so rk $K_{0}(\mathrm{~T})=r$. If $\mathrm{T}$ has the Serre functor $S$ in the sense of Bondal and Kapranov [6], then $S$ induces an automorphism $\mathfrak{s}$ of $K_{0}(\mathrm{~T})$.

Theorem 3.1. Let $\mathrm{D}^{\mathrm{b}}(\operatorname{coh} X)$ be the bounded derived category of coherent sheaves on a smooth projective variety $X$.

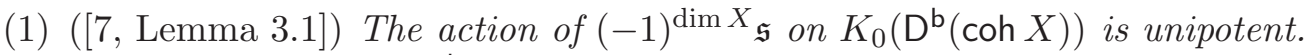

(2) ([2, Corollary 25]) If $\mathrm{D}^{\mathrm{b}}(\operatorname{coh} X)$ admits a full strong exceptional sequence, then

$$
\chi\left(\mathrm{HH}^{\bullet}(X)\right)=(-1)^{\operatorname{dim} X} \operatorname{rk} K_{0}\left(\mathrm{D}^{\mathrm{b}}(\operatorname{coh} X)\right) .
$$

where $\chi\left(\mathrm{HH}^{\bullet}(X)\right):=\sum_{i \in \mathbb{Z}}(-1)^{i} \operatorname{dim}_{k} \mathrm{HH}^{i}(X)$.

Let $A=A(\alpha, \beta)$ be a graded down-up algebra with $\operatorname{deg} x=1, \operatorname{deg} y=n \geq 1$, and $\beta \neq 0$. Then $\mathrm{D}^{\mathrm{b}}$ (tails $A$ ) has a full strong exceptional sequence of length $2 n+2$ by [20, Propositions 4.3, 4.4], so rk $K_{0}\left(\mathrm{D}^{\mathrm{b}}(\right.$ tails $\left.A)\right)=2 n+2$. Moreover $\mathrm{D}^{\mathrm{b}}($ tails $A)$ has the Serre functor by [21, Appendix A]. Note that $\operatorname{gldim}(\operatorname{tails} A)=\operatorname{gldim} \nabla A=2$.

If $n=1$, then $\mathfrak{s}$ acts unipotently on $K_{0}\left(\mathrm{D}^{\mathrm{b}}\right.$ (tails $\left.A\right)$ ) (see [2, comments after Remark 26]), and it follows from Theorem 1.3 that

$$
\chi\left(\mathrm{HH}^{\bullet}(\nabla A)\right)=4=\operatorname{rk} K_{0}\left(\mathrm{D}^{\mathrm{b}}(\text { tails } A)\right)
$$

where $\chi\left(\mathrm{HH}^{\bullet}(\nabla A)\right):=\sum_{i \in \mathbb{Z}}(-1)^{i} \operatorname{dim}_{k} \mathrm{HH}^{i}(\nabla A)$, so an analogue of Theorem 3.1 holds. For the case $n \geq 2$, we have the following. 
Proposition 3.2. If $n=2$, then $\mathfrak{s}$ acts unipotently on $K_{0}\left(\mathrm{D}^{\mathrm{b}}(\right.$ tails $\left.A)\right)$ and

$$
\chi\left(\mathrm{HH}^{\bullet}(\nabla A)\right)=6=\mathrm{rk} K_{0}\left(\mathrm{D}^{\mathrm{b}}(\operatorname{tails} A)\right) .
$$

If $n \geq 3$, then $\mathfrak{s}$ does not act unipotently on $K_{0}\left(\mathrm{D}^{\mathrm{b}}(\right.$ tails $\left.A)\right)$ and

$$
\chi\left(\mathrm{HH}^{\bullet}(\nabla A)\right)=n+2 \neq 2 n+2=\operatorname{rk} K_{0}\left(\mathrm{D}^{\mathrm{b}}(\text { tails } A)\right) .
$$

Proof. First, (3.1) and (3.2) follow from Theorem 1.4. If $n=2$, then the Gram matrix of $K_{0}\left(\mathrm{D}^{\mathrm{b}}(\operatorname{tails} A)\right)$ is given by

$$
M=\left(\begin{array}{llllll}
1 & 1 & 2 & 3 & 4 & 5 \\
0 & 1 & 1 & 2 & 3 & 4 \\
0 & 0 & 1 & 1 & 2 & 3 \\
0 & 0 & 0 & 1 & 1 & 2 \\
0 & 0 & 0 & 0 & 1 & 1 \\
0 & 0 & 0 & 0 & 0 & 1
\end{array}\right)
$$

so one can verify that

$$
\mathfrak{s}=M^{-1} M^{T}=\left(\begin{array}{cccccc}
7 & 5 & 4 & 3 & 2 & 1 \\
1 & 2 & 1 & 1 & 1 & 1 \\
-5 & -4 & -2 & -2 & -1 & 0 \\
-6 & -5 & -4 & -2 & -2 & -1 \\
-1 & -1 & -1 & -1 & 0 & -1 \\
5 & 4 & 3 & 2 & 1 & 1
\end{array}\right)
$$

is unipotent. We now consider the case $n \geq 3$. Suppose that $\mathfrak{s}$ acts unipotently on $K_{0}\left(\mathrm{D}^{\mathrm{b}}(\operatorname{tails} A)\right)$. Then $\mathfrak{s}=M^{-1} M^{T}$ is unipotent where $M$ is the Gram matrix. Since $M$ coincides with the Cartan matrix of $\nabla A$, the Coxeter matrix $\Phi_{\nabla A}$ of $\nabla A$ is obtained as $-M^{-T} M$ (see [12, Section 1]), so we have

$$
-\operatorname{tr} \Phi_{\nabla A}=\operatorname{tr} M^{-T} M=\operatorname{tr} M^{-1} M^{T}=\operatorname{tr} \mathfrak{s} .
$$

Unipotency of $\mathfrak{s}$ implies $\operatorname{tr} \mathfrak{s}=2 n+2$, so it follows that $-\operatorname{tr} \Phi_{\nabla A}=2 n+2$. By Happel's trace formula [12, we have $\chi\left(\mathrm{HH}^{\bullet}(\nabla A)\right)=-\operatorname{tr} \Phi_{\nabla A}=2 n+2$, but this contradicts (3.2). Hence $\mathfrak{s}$ does not act unipotently.

In respect of Proposition 3.2, when $n=2, \mathrm{D}^{\mathrm{b}}$ (tails $A$ ) behaves a bit like a geometric object (a smooth projective surface), but when $n \geq 3, \mathrm{D}^{\mathrm{b}}$ (tails $A$ ) is not equivalent to the derived category of any smooth projective surface.

\section{ACKNOWLEDGMENTS}

The authors are grateful to Teruyuki Yorioka for his support and helpful discussions. They also thank the referee for useful comments in improving the paper. The first author was supported by JSPS Grant-in-Aid for Early-Career Scientists 18K13397. The second author was supported by JSPS Grant-in-Aid for Early-Career Scientists 18K13381.

\section{REFERENCES}

[1] M. Artin and J. J. Zhang, Noncommutative projective schemes, Adv. Math. 109 (1994), no. 2, 228-287.

[2] P. Belmans, Hochschild cohomology of noncommutative planes and quadrics, J. Noncommut. Geom. 13 (2019), no. 2, 769-795.

[3] G. Benkart, Down-up algebras and Witten's deformations of the universal enveloping algebra of $\mathfrak{s l}_{2}$, in Recent progress in algebra (Taejon/Seoul, 1997), Contemp. Math., vol. 224, Amer. Math. Soc., Providence, RI, 1999, pp. $29-45$.

[4] G. Benkart and T. Roby, Down-up algebras, J. Algebra 209 (1998), no. 1, 305-344. Addendum: J. Algebra 213 (1999), no. 1, 378.

[5] G. Benkart and S. Witherspoon, A Hopf structure for down-up algebras, Math. Z. 238 (2001), no. 3, $523-553$.

[6] A. I. Bondal and M. M. Kapranov, Representable functors, Serre functors, and reconstructions, Math. USSR-Izv. 35 (1990), no. 3, 519-541.

[7] A. I. Bondal and A. E. Polishchuk, Homological properties of associative algebras: the method of helices, Russian Acad. Sci. Izv. Math. 42 (1994), no. 2, 219-260. 
[8] S. Chouhy, E. Herscovich, and A. Solotar, Hochschild homology and cohomology of down-up algebras, J. Algebra 498 (2018), 102-128.

[9] P. A. A. B. Carvalho and I. M. Musson, Down-up algebras and their representation theory, J. Algebra 228 (2000), no. $1,286-310$.

[10] E. L. Green and N. Snashall, Projective bimodule resolutions of an algebra and vanishing of the second Hochschild cohomology group, Forum Math. 16 (2004), 17-36.

[11] D. Happel, Hochschild cohomology of finite-dimensional algebras, in Séminaire d'Algèbre Paul Dubreil et Marie-Paul Malliavin, 39ème Année (Paris, 1987/1988), Lecture Notes in Math., vol. 1404, Springer, Berlin, 1989, pp. $108-126$.

[12] D. Happel, The trace of the Coxeter matrix and Hochschild cohomology, Linear Algebra Appl. 258 (1997), $169-177$.

[13] M. Herschend, O. Iyama, and S. Oppermann, n-Representation infinite algebras, Adv. Math. 252 (2014), $292-342$.

[14] E. Kirkman and J. Kuzmanovich, Fixed subrings of noetherian graded regular rings, J. Algebra 288 (2005), no. 2, 463-484.

[15] E. Kirkman, J. Kuzmanovich, and J. J. Zhang, Invariant theory of finite group actions on down-up algebras, Transformation Groups 20 (2015), no. 1, 113-165.

[16] E. Kirkman, I. Musson, and D. Passman, Noetherian down-up algebras, Proc. Amer. Math. Soc. 127 (1999), no. 11, 3161-3167.

[17] O. Lezama, Y.-H. Wang, and J. J. Zhang, Zariski cancellation problem for non-domain noncommutative algebras Math. Z. 292 (2019), no. 3-4, 1269-1290.

[18] W. Lowen and M. Van den Bergh, Hochschild cohomology of abelian categories and ringed spaces, Adv. Math. 198 (2005), no. 1, 172-221.

[19] W. Lowen and M. Van den Bergh, Deformation theory of abelian categories, Trans. Amer. Math. Soc. 358 (2006), no. $12,5441-5483$.

[20] H. Minamoto and I. Mori, The structure of AS-Gorenstein algebras, Adv. Math. 226 (2011), no. 5, 4061-4095.

[21] K. de Naeghel and M. Van den Bergh, Ideal classes of three-dimensional Sklyanin algebras, J. Algebra 276 (2004), no. $2,515-551$.

[22] C. A. Weibel, An introduction to homological algebra, Cambridge Studies in Advanced Mathematics, vol. 38. Cambridge University Press, Cambridge, 1994.

[23] K. Zhao, Centers of down-up algebras, J. Algebra 214 (1999), no. 1, 103-121.

Department of Mathematics, Faculty of Science, Tokyo University of Science, 1-3 Kagurazaka, ShinJYUKU, TOKYO, 162-8601, JAPAN

E-mail address: itaba@rs.tus.ac.jp

Department of Mathematics, Faculty of Education, Hirosaki University, 1 Bunkyocho, Hirosaki, AoMORI, 036-8560, JAPAN

E-mail address: k-ueyama@irosaki-u.ac.jp 